\title{
Mutación R249S TP53 en pacientes con cirrosis y carcinoma hepatocelular en un hospital de Medellín
}

\author{
TP53 R249S mutation in patients with cirrhosis and \\ hepatocellular carcinoma in a hospital at Medellin
}

Melissa Montoya-Guzmán', Alejandra Duque-Jaramillo², Marcela Gaviria-Calle ${ }^{3}$ vrAc, Sergio Hoyos", Juan Carlos Restrepo5, María Cristina Navas ${ }^{6}$ CvLAC

Fecha correspondencia: Recibido: mayo 6 de 2018. Revisado: octubre 1 de 2018. Aceptado: marzo 27 de 2019.

Forma de citar:

Montoya-Guzmán M, DuqueJaramillo A, Gaviria-Calle M, Hoyos S, Restrepo JC, Navas MC. Mutación R249S TP53 en pacientes con cirrosis y carcinoma hepatocelular en un hospital de Medellín. Rev CES Med 2019; 33(2): 100-110.

\section{Open access}

(c) Derecho de autor

Licencia creative commons

Ética de publicaciones

Revisión por pares

Gestión por Open Journal System

DOI: http://dx.doi.org/10.21615/

cesmedicina.33.2.3

ISSN 0120-8705

e-ISSN 2215-9177

\section{Resumen}

Introducción: la exposición dietaria a la aflatoxina es un factor de riesgo para carcinoma hepatocelular, el cáncer primario de hígado más frecuente. Esta asociación se estableció gracias a la evidencia in vitro e in vivo de la relación entre la exposición a la aflatoxina B1 y la transversión $\mathrm{G} \rightarrow$ T en el codón 249 del gen TP53, así como evidencia de la sinergia entre la aflatoxina y la infección crónica por virus de la hepatitis B. Métodos: se determinó la frecuencia de la mutación R249S del gen TP53 en 30 pacientes con diagnóstico de cirrosis y/o carcinoma hepatocelular quienes fueron sometidos a trasplante hepático en un hospital en Medellín, Colombia. Se extrajo ADN a partir de las muestras de explante hepático, se amplificó el fragmento de interés y se detectó la mutación por polimorfismos de longitud de fragmentos de restricción. Resultados: se encontró la mutación R249S en una de las 30 muestras analizadas (3,33 \%) y se determinó, por medio de marcadores serológicos, infección por el virus de la hepatitis B en dos casos $(6,67 \%)$. No se encontró simultáneamente la mutación y la presencia de los marcadores de infección por virus de la hepatitis B. Conclusión: los resultados sugieren una baja exposición dietaria con aflatoxina B1 en la población de estudio. Sin embargo, es importante tener en cuenta la regulación de los límites permisibles de aflatoxina B1 y la inclusión en el diagnóstico diferencial de carcinoma hepatocelular, dada la heterogeneidad de las condiciones de la población en diferentes regiones del país.

Palabras claves: Carcinoma hepatocelular; Aflatoxina B1; TP53; Virus de la hepatitis B.

\section{Abstract}

Introduction: The dietary exposure to aflatoxin is a risk factor of hepatocellular carcinoma, the most frequent primary liver cancer. This risk factor was identified after in vivo and in vitro evidence of the relation between exposure to aflatoxin B1 and transversion $G \rightarrow T$ at 249 codon of the TP53 gene; as well as evidence of the synergy between hepatitis $B$ virus chronic infection Methods: the frequency of the R249S mutation of the TP53 gene was determined in 30 cases of cirrhosis and/or hepatocellular carcinoma, 


\section{Sobre los autores:}

1. Estudiante de Biología, Facultad de Ciencias y Biotecnología, Programa de Biología, Universidad CES. Grupo de Gastrohepatología, Facultad de Medicina, Universidad de Antioquia.

2. Joven investigadora. Grupo de Gastrohepatología, Facultad de Medicina, Universidad de Antioquia.

3. MSc. Grupo de Gastrohepatología, Facultad de Medicina, Universidad de Antioquia.

4. MSc. Profesor titular. Grupo de Gastrohepatología, Facultad de Medicina, Universidad de Antioquia. Médico especialista Hospital Pablo Tobón Uribe.

5. PhD. Profesor titular. Grupo de Gastrohepatología, Facultad de Medicina, Universidad de Antioquia. Médico especialista Hospital Pablo Tobón Uribe.

6. PhD. Profesora titular, Facultad de Medicina, Coordinadora Grupo de Gastrohepatología, Universidad de Antioquia. maria.navas@udea.edu.co with liver transplantation in the hepatology unit of a hospital in Medellín, Colombia. DNA was extracted from the liver explant samples; the sequence of interest was amplified, and the mutation was detected by restriction fragment length polymorphisms. Results: the R249S mutation was found in 1 of the 30 samples analyzed (3.33\%); and hepatitis B virus infection was detected by serological markers in 2 of the 30 cases $(6.67 \%)$. We did not find the mutation and the presence of hepatitis $B$ virus infection markers at the same time in any of the samples. Conclusion: The results suggest a low dietary exposure with aflatoxin B1 in the study population. However, it is important to take into consideration the regulation of the permissible limits of aflatoxin B1 and the inclusion in the differential diagnosis of hepatocellular carcinoma, given the heterogeneity of the conditions of the population in different regions of the country.

Key words: Hepatocellular carcinoma; Aflatoxin B1; TP53; Hepatitis B Virus.

\section{Introducción}

El carcinoma hepatocelular representa el 80 \% de casos de cáncer primario de hígado, y es la segunda causa de muerte más frecuente asociada al cáncer (1). Sus principales factores de riesgo son la infección crónica por el virus de la hepatitis B (VHB), la infección crónica por el virus de la hepatitis C, el consumo crónico de alcohol, el síndrome metabólico y la exposición dietaria a aflatoxina B1 (AFB1) (2).

La aflatoxina B1 es un metabolito secundario producido por hongos, principalmente por especies del género Aspergillus. En los trópicos, debido a las condiciones de humedad y temperatura, se facilita su proliferación en la cadena de producción y almacenamiento de alimentos, contaminando granos, nueces y semillas (3).

La aflatoxina B1 es un potente agente genotóxico, clasificado en el grupo 1 de cancerígenos por la Agencia Internacional para la Investigación del Cáncer (1). Al consumir alimentos contaminados con aflatoxina B1, esta micotoxina es metabolizada en el hígado por el complejo citocromo CYP450, dando lugar a 8,9-exo-epóxido, altamente reactivo, que se une covalentemente con proteínas (aflatoxicosis) o al ADN, formando aductos (ㄴ).

El epóxido tiene una marcada preferencia por el gen TP53, específicamente por el exón 7 codón 249, causando la transversión $G \rightarrow T$, que conlleva al cambio de arginina por serina en la proteína (드). TP53 es un gen supresor de tumores que codifica la proteína p53, un factor de transcripción que regula el ciclo celular, la muerte celular programada, la respuesta al estrés celular y la reparación del ADN (ㅁ).

En poblaciones con alta exposición a aflatoxinas, como países tropicales y subtropicales, se ha encontrado la mutación R249S del gen TP53 en 30 \% a 60 \% de los pacientes con carcinoma hepatocelular (7). En individuos sin evidencia de enfermedad crónica hepática o asintomáticos, la frecuencia de la mutación varía según la presencia del antígeno de superficie del virus de la hepatitis B (HBsAg), con su máximo nivel en pacientes positivos para este antígeno viral (8).

Se ha demostrado en modelos in vivo e in vitro la relación causal de aflatoxina B1 y R249S (2,7), razón por la cual se ha descrito que en pacientes con carcinoma hepatocelular esta mutación sirve como una "huella" de exposición a aflatoxina B1, lo cual está sustentado bajo el criterio de Bradford-Hill (9). 
Se ha descrito una sinergia entre la exposición dietaria a aflatoxinas y la infección crónica por el virus de la hepatitis $B$ en el riesgo de carcinoma hepatocelular.
El estudio de este factor de riesgo es de importancia en salud pública, con el fin de regular el nivel máximo permisible de aflatoxinas presente en alimentos de consumo humano por la legislación del país y la inclusión en el diagnóstico diferencial en pacientes a riesgo de cáncer primario de hígado.

Adicionalmente, se ha descrito una sinergia entre la exposición dietaria a aflatoxinas y la infección crónica por el virus de la hepatitis B en el riesgo de carcinoma hepatocelular $(10,11)$. Se ha postulado que la sinergia de la infección por VHB y la exposición a aflatoxina B1 en el desarrollo de carcinoma hepatocelular se debe principalmente a la función pleiotrópica de la proteína viral X, que puede interferir con la reparación del ADN celular (10).

La relación entre la infección crónica por virus de la hepatitis $\mathrm{B}$ y la exposición a aflatoxina B1 se ha documentado en otras investigaciones que reportan un Odds Ratio de 399 (IC 95 \%: 48,6 - 3 270) entre los casos de carcinoma hepatocelular positivos para la mutación e infección por VHB y el grupo control (12). Asimismo, se ha encontrado esta sinergia en modelos animales como la marmota y ratones transgénicos infectados con VHB $(\underline{13}, \underline{14})$.

Este trabajo de investigación se enmarca en un estudio de casos y controles en el que se evaluaron los factores de riesgo de las hepatopatías terminales en población colombiana.

Se planteó este estudio para determinar la frecuencia de la mutación R249S del gen TP53 como evidencia de la exposición a aflatoxina B1.

\section{Metodología}

\section{Tejido hepático}

Se recolectaron 30 muestras de explante de pacientes con diagnóstico de cirrosis y/o carcinoma hepatocelular, sometidos a trasplante hepático en el Hospital Pablo Tobón Uribe de Medellín, desde junio de 2014 hasta agosto de 2016; las muestras se almacenaron $\mathrm{a}-70^{\circ} \mathrm{C}$.

La extracción de ADN total con TRIzol (Ambion ${ }^{\circ}$, USA) se realizó a partir de tejido hepático siguiendo las instrucciones de la casa comercial. El ADN se re-suspendió en Hepes comercial (Amresco , USA) y se almacenó a $-20{ }^{\circ} \mathrm{C}$. Para determinar la pureza del ADN extraído se cuantificaron los radios 260/280 y 260/230 (NanoDrop 2000 Thermo Fisher Scientific, USA). Luego se prepararon diluciones de ADN para tener una concentración de trabajo estándar de $40 \mathrm{ng} / \mu \mathrm{L}$. Posteriormente, se verificó la integridad del ADN extraído mediante una PCR del gen constitutivo gliceraldehído-3-fosfato deshidrogenasa con los primers 5'-CCTTCATTGACCTCAACTACATGG-3' y 5'-AGTCTTCTGGGTGGCAGTGATGG-3' (15).

Los ensayos de amplificación del fragmento de interés de TP53 se realizaron utilizando los primers 5'-CTTGCCACAGGTCTCCCCAA-3' (nt 13 941-13 960) y 5'-AGGGGTCAGCGGCAAGCAGA-3' (nt 14 167-14 158) (12). El volumen final de la reacción fue de $25 \mu \mathrm{L}$ utilizando $200 \mathrm{ng}$ de ADN, $1 \mathrm{U}$ de Taq ADN polimerasa (Thermo Fisher Scientific ${ }^{\circ}$, USA), 0,6 $\mu \mathrm{M}$ de cada primer, $200 \mu \mathrm{M}$ de cada dNTP (Fermentas ${ }^{\circ}$, Canadá), 2,5 $\mu \mathrm{L}$ de 10 X Buffer Taq polimerasa (Thermo Fisher Scientific ${ }^{\circ}$, USA), 1,5 mM de MgSO4 (Thermo Fisher Scientific ${ }^{\circ}$, USA) y agua libre de nucleasas (Amresco $0^{\circ}$, USA). El ciclaje se estandarizó con el siguiente protocolo: 4 min a $94^{\circ} \mathrm{C}$ para la activación 
Se recolectaron 30 muestras de explante de pacientes con diagnóstico de cirrosis y/o carcinoma hepatocelular. de la polimerasa, posteriormente 35 ciclos a $94^{\circ} \mathrm{C}$ por $30 \mathrm{~s}, 65^{\circ} \mathrm{C}$ por $30 \mathrm{~s}, 72{ }^{\circ} \mathrm{C}$ por $30 \mathrm{~s}$, y finalmente $7 \mathrm{~min}$ a $72{ }^{\circ} \mathrm{C}$ para la extensión final (iCycler, Biorad).

En todos los ensayos de reacción en cadena de polimerasa se incluyó un control negativo (agua) y como control positivo ADN de la línea celular derivada de hepatoma humano PLC/PRF/5 (ATTC \# CRL-8024), homocigota para la mutación R249S. Los resultados se revelaron mediante electroforesis en un gel de agarosa al 1,5\% corrido a $100 \mathrm{~V}$ por una hora, con un fragmento esperado de $237 \mathrm{pb}$.

Los productos de la reacción en cadena de polimerasa se sometieron a un ensayo de polimorfismos de longitud de fragmentos de restricción (por sus siglas en inglés, RFLP) con la enzima Haelll (Invitrogen", USA) que reconoce GG $\downarrow C C$ del codón 249 (AGG). El control positivo para este ensayo fue el producto de reacción en cadena de polimerasa de la línea celular PLC/PRF/5. Para la reacción fueron necesarios $5 \mu \mathrm{L}$ de producto de PCR, $2 \mu \mathrm{L}$ de 10 X Buffer M (Invitrogen ${ }^{\circ}$, USA) y $5 \mathrm{U}$ de HaellI (Invitrogen ${ }^{\oplus}$, USA). Los resultados se revelaron mediante electroforesis en un gel de agarosa al $3 \%$ con cinco fragmentos esperados de 92, 66, 37, 30 y 12 pb para las muestras wildtype y cuatro fragmentos para las mutantes R249S, de 158, 37, 30 y 12 pb (Figura 1A). Tanto para la reacción en cadena de polimerasa del gen TP53 como la RFLP se realizaron duplicados para verificar los resultados.

Para describir y analizar las variables se usaron estadísticos descriptivos; se verificó el equilibrio de Hardy Weinberg y la posible asociación entre la mutación puntual en el codón 249 del gen TP53 y la infección por VHB se verificó usando una prueba exacta de Fisher. Los análisis se llevaron a cabo en el programa R usando un valor de significancia $p<0,05$.

El trabajo fue aprobado por el comité de bioética del Instituto de Investigaciones Médicas de la Universidad de Antioquia y el comité de ética del Hospital Pablo Tobón Uribe. Todos los participantes firmaron un consentimiento informado en el que autorizaron que las muestras de sangre o tejido hepático fueran utilizadas para el análisis de factores de riesgo de cirrosis y cáncer de hígado con métodos bioquímicos y de biología molecular; además del polimorfismo en algunos genes que codifican enzimas que participan en el metabolismo del alcohol como alcohol deshidrogenasa y el citocromo P450 CYP2E1. Adicionalmente, los pacientes respondieron una encuesta de datos sociodemográficos.

\section{Resultados}

La mayoría de los pacientes fueron del sexo masculino (60 \%), con una edad promedio de 56 años (IC $95 \%$ : 51,5 - 60,7). Otros datos demográficos y clínicos de los pacientes se presentan en el cuadro 1. No se encontraron diferencia entre las variables sobrepeso/no-sobrepeso ( $p=0,85)$, así como tampoco entre la zona de nacimiento urbana/ rural $(p=0,85)$. Adicionalmente se crearon dos grupos, del estrato 1-3 y del 4-6. Tampoco se encontró relación entre la mutación y el estrato socioeconómico $(p=0,33)$.

La mutación puntual R249S del gen TP53 fue detectada en un solo caso (3,33 \%) proveniente de un paciente con cirrosis y carcinoma hepatocelular (figura 1B). La población se ajustó al equilibrio de Hardy Weinberg $(p=0,926)$. Adicionalmente, se encontró infección crónica por virus de hepatitis B en 6,67 \% (dos casos), ambos positivos para HBsAg y anti-HBc total y negativos para la mutación R249S. La distribución de la mutación en los casos evaluados no mostró una relación de dependencia con el factor etiológico de infección crónica por VHB $(p=1)$. 
La mutación puntual R249S del gen TP53 fue detectada en un solo caso $(3,33 \%)$ proveniente de un paciente con cirrosis y carcinoma hepatocelular.
Cuadro 1. Datos demográficos y clínicos de la población de estudio

\begin{tabular}{lc}
\hline Variable & $\%(\boldsymbol{n})$ \\
\hline Rango de edad & $22-78$ \\
Sexo femenino & 40 \\
Sexo masculino & 60 \\
Lugar de nacimiento zona rural & 46,7 \\
Lugar de nacimiento zona urbana & 53,3 \\
Sobrepeso & 46,7 \\
\hline Diagnóstico & \\
\hline Cirrosis & 75 \\
Carcinoma hepatocelular & 3,6 \\
Cirrosis y carcinoma hepatocelular & 21,43 \\
R249S Positivo & 3,3 \\
VHB Positivo & 6,7 \\
\hline
\end{tabular}

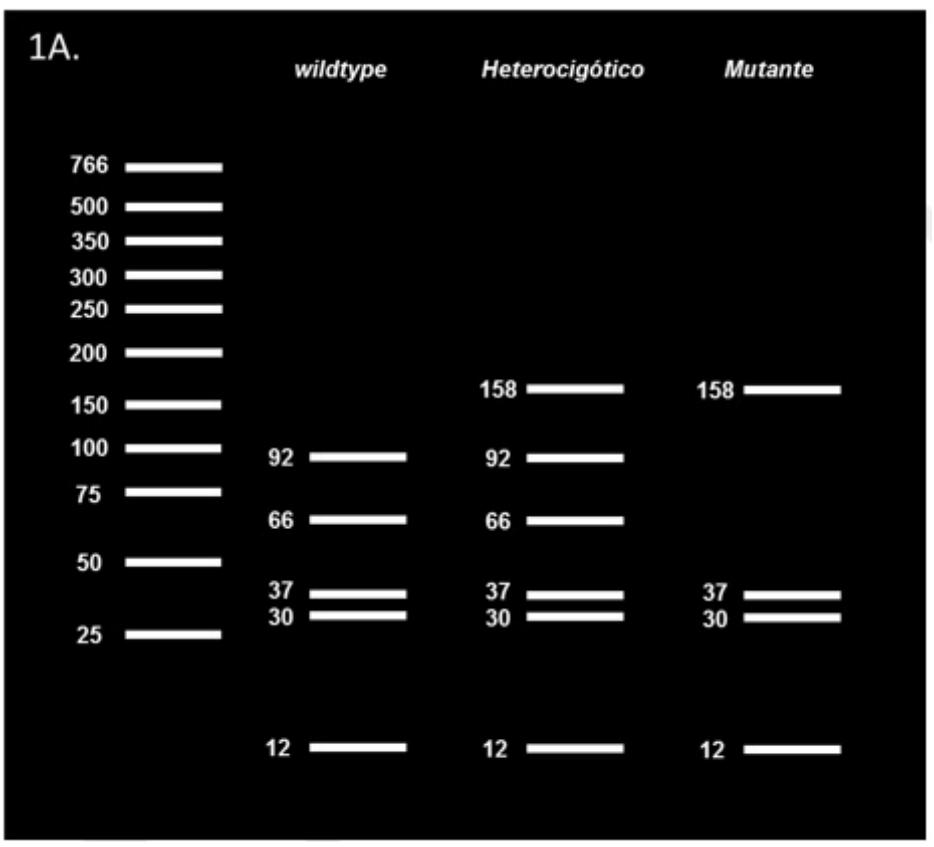

Figura 1A. Patrón de bandas esperadas para la RFLP de la mutación R249S del gen TP53.

En la figura 1A se muestra un esquema con los cuatro puntos de cortes esperado ( 5 bandas) para el genotipo wildtype, tres puntos de corte esperado ( 4 bandas) para el mutante y la combinación de ambos alelos en el genotipo heterocigótico para un total de 6 bandas. 
Mayo - agosto de 2019 - Pág 105

Evidencias in vivo e in vitro demuestran que la mutación R249S del gen TP53 es uno de los principales cambios genéticos en el carcinoma hepatocelular inducido por esta micotoxina.

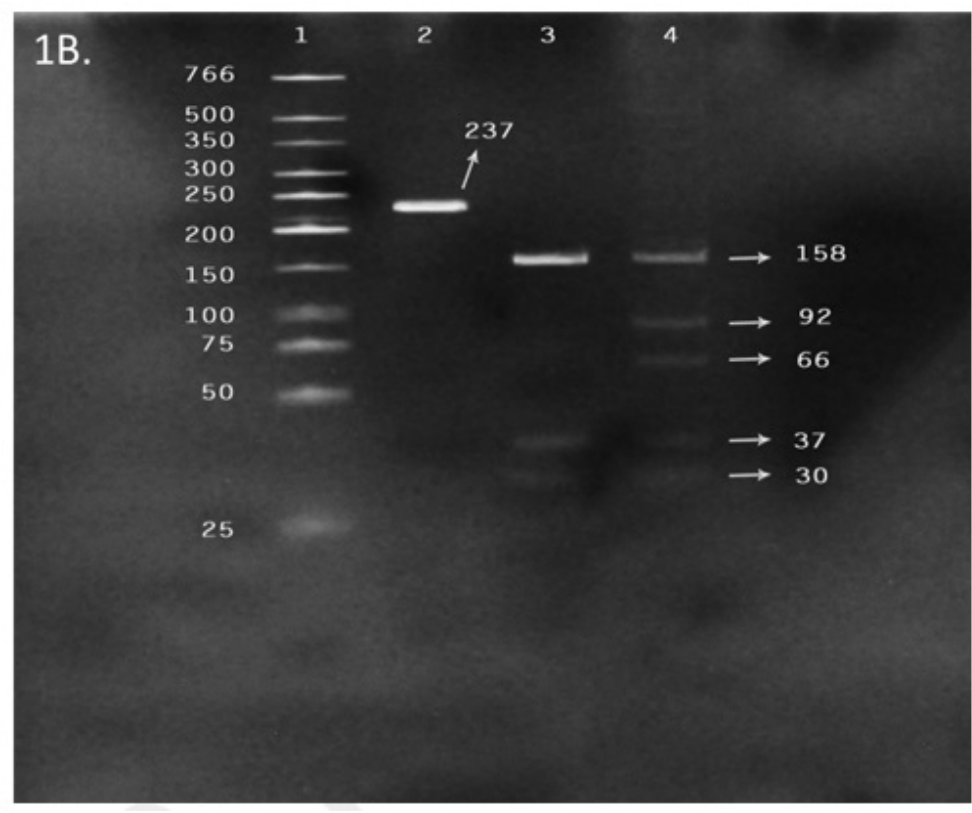

Figura 1B. Detección de la mutación R249S del gen TP53 por RFLP en muestras de tejido hepático obtenidas de pacientes con diagnóstico de cirrosis y/o carcinoma hepatocelular

Línea 1, marcador de peso molecular de 25pb. Línea 2, control negativo con ADN de HepG2 sin enzima. Línea 3, control positivo con la línea celular PLC/ PRF/5 homocigótica para la mutación, con tres puntos de corte en cada alelo (se observan 3 bandas). Línea 4, muestra HPTU-01-062 heterocigótica para la mutación, con cuatro puntos de cortes (se observan 4 bandas) para el alelo mutante y tres puntos de corte en el alelo wildtype (se observan 3 bandas). En las líneas 3 y 4 no se logra observar la banda de 12 pb debido a las características del gel.

\section{Discusión}

La aflatoxina B1 es reconocida como un potente hepatocarcinógeno. Evidencias in vivo e in vitro demuestran que la mutación R249S del gen TP53 es uno de los principales cambios genéticos en el carcinoma hepatocelular inducido por esta micotoxina $(16,17)$. En zonas de alta exposición como Guangxi, China, Stern et al. encuentran la mutación R249S en el 36 \% de las muestras (18/50) obtenidas de pacientes con diagnóstico de carcinoma hepatocelular (18). Adicionalmente, Stern et al. realizan un metaanálisis con otros 48 reportes de esta zona geográfica y demuestran que la proporción de casos con la mutación R249S se correlaciona con el nivel de exposición dietaria a aflatoxinas (18).

Para Tailandia se ha descrito una frecuencia de la mutación de $26,9 \%$ en casos de carcinoma hepatocelular (19). Este resultado puede relacionarse con la dieta, puesto que, en dos de los principales alimentos de la población tailandesa, maíz y nueces, se han encontrado concentraciones de aflatoxina B1 superiores a los $20 \mu \mathrm{g} / \mathrm{kg}$ permitidas por el gobierno de este país $(\underline{19}, \underline{20})$.

En el presente estudio la población se encontró en equilibrio de Hardy Weinberg, lo cual sugiere que no hay estructuración poblacional (21). La infección por VHB se demostró en dos de los casos, que no fueron positivos para la mutación R249S $(\underline{22}, \underline{23})$. La frecuencia de la mutación detectada por PCR-RFLP es similar a la reportada en el metaanálisis de Liu et al. (3 \%) en el que se estiman los casos de carcinoma hepatocelular atribuibles a exposición a aflatoxinas en países de África, Norte América, 
En Colombia se ha reportado contaminación por aflatoxina B1 en alimentos provenientes de diferentes zonas del país.
Latino América, Mediterráneo, Asia y Europa, según los datos de contaminación en alimentos, consumo de alimentos contaminados y prevalencia de la infección por virus de la hepatitis B (24).

En un estudio realizado con muestras de carcinoma hepatocelular remitidas a laboratorios de patología de Bogotá, Medellín y Cali durante el periodo 2000 a 2007, se encuentra la mutación R249S por PCR-RFLP y secuenciación en 10,5 \% (4/38) de las muestras de tejido hepático embebido en parafina (25). Dos de los casos analizados en este estudio fueron positivos para R249S e infección por VHB simultáneamente.

Estos hallazgos sugieren que la exposición a aflatoxina B1 en Colombia es entre baja e intermedia. La frecuencia encontrada en dicho estudio es mayor que en el presente estudio, debido, posiblemente, a que se incluyeron pacientes de varias regiones del país con diferencias en la prevalencia de infección por VHB y el nivel de contaminación dietaria por aflatoxinas.

Otros estudios demuestran la exposición a aflatoxina B1 en pacientes con hepatopatías terminales en México y Brasil. Soini et al. encuentran la mutación R249S por secuenciación en 18,7 \% (3/16) de muestras de tejido hepático obtenido de pacientes mexicanos con diagnóstico de carcinoma hepatocelular (26). En Brasil, Carvalho et al. analizan 33 muestras de pacientes con carcinoma hepatocelular y 43 de pacientes con cirrosis. La mutación R249S se demostró en 39 \% de las muestras por la técnica de PCR-RFLP y en $14,6 \%$ de las muestras por la técnica de secuenciación (27). Nogueira et al. analizan muestras de carcinoma hepatocelular obtenidas de 74 pacientes de la ciudad de São Paulo. La mutación se encontró por PCR-RFLP en el $28 \%$ de las muestras y por secuenciación en $16,2 \%$, lo cual sugiere que existe una contaminación moderada en Brasil y que la aflatoxina B1 cumple un rol en los casos de carcinoma hepatocelular en este país (22).

En Colombia, se ha reportado contaminación por aflatoxina B1 en alimentos provenientes de diferentes zonas del país como: Pamplona, Norte de Santander, con niveles entre 18,42 y $71,25 \mu \mathrm{g} / \mathrm{kg}$ en alimentos de consumo infantil comercializados en la zona (28); además, cinco departamentos de la costa Caribe con reportes de niveles entre 2,4 y 12,5 ppb en arroz y maíz colectadas en diferentes fábricas y comercializadoras (29). En el departamento de Amazonas se encontró aflatoxina B1 en niveles entre 6,4 y $458,2 \mu \mathrm{g} / \mathrm{kg}$ en muestras de maíz, arroz y mandioca, recolectadas en tres comunidades indígenas (30).

Según la legislación colombiana el nivel de aflatoxinas B1, B2, G1 y G2 para todos los cereales y productos a base de cereales, incluidos los productos transformados, no debe ser mayor de $4 \mu \mathrm{g} / \mathrm{kg}$ y el nivel máximo permitido de aflatoxinas B1, B2, G1 y G2 en maíz para el consumo humano es de $10 \mu \mathrm{g} / \mathrm{kg}$ (31). Sin embargo, para este último se dispuso que "dicho nivel máximo no es posible en maíz de producción nacional dadas algunas condiciones de manejo postcosecha", por lo cual mediante la resolución 02671 de 2014 se aumentaron los límites para el maíz a 20 mg/kg y el arroz a $10 \mu \mathrm{g} / \mathrm{kg}$ (32).

Una alternativa para controlar la contaminación dietaria con aflatoxinas es la sugerida por Wu et al. quienes demuestran que la prevención por biocontrol en los cultivos e intervenciones postcosecha tienen un radio costo-efectivo de 5,10-24,8 y 0,21-2,08, respectivamente (33). 
La exposición dietaria a aflatoxinas y la infección crónica por el virus de la hepatitis B son dos importantes factores de riesgo para el carcinoma hepatocelular que deben preocupar a las autoridades de salud pública.
La contaminación dietaria por aflatoxinas es un fenómeno que depende de las condiciones ambientales, manejo adecuado, cadena de distribución y fuente de abastecimiento de alimentos (3). Algunas de estas características podrían variar entre los diferentes estratos socioeconómicos en el país. Pese a esto, no se pudo observar una relación significativa entre la mutación y el estrato. La falta de significancia en las diferentes pruebas realizadas puede deberse al limitado tamaño de muestra y a que en poblaciones con baja exposición a aflatoxinas y baja prevalencia de infección por VHB esta mutación es reparada por escisión de nucleótidos (NER) $(\underline{3}, \underline{34})$.

La exposición dietaria a aflatoxinas y la infección crónica por el virus de la hepatitis B son dos importantes factores de riesgo para el carcinoma hepatocelular que deben preocupar a las autoridades de salud pública. Sin embargo, la frecuencia de la mutación en el codón 249ser del gen TP53 como marcador de la posible exposición previa a aflatoxinas y la posible relación epidemiológica con VHB y el CHC ha sido poco explorada en Colombia.

Teniendo en cuenta los resultados de este estudio y la evidencia de contaminación con aflatoxina B1 de alimentos para el consumo humano en Colombia se hace necesario sugerir inspecciones sanitarias, regulación de los límites permisibles por la legislación del país y la inclusión en el diagnóstico diferencial de carcinoma hepatocelular, dada la heterogeneidad de las condiciones de la población en diferentes regiones del país.

\section{Conflicto de intereses}

Los autores no declaran conflicto de intereses.

\section{Financiación}

Este proyecto fue financiado por el Departamento Administrativo de Ciencia, Tecnología e Innovación, Colciencias. Código del proyecto: 111556935008.

\section{Bibliografía}

1. Stewart BW, Wild C, International Agency for Research on Cancer, World Health Organization, editores. World cancer report 2014. Lyon, France: International Agency for Research on Cancer; 2014. 630 p.

2. McGlynn KA, London WT. The global epidemiology of hepatocellular carcinoma: present and future. Clin Liver Dis. 2011;15(2):223-243, vii-x.

3. Venegas AC, Guerra JJH, Navas MCN. Exposición a aflatoxina: un problema de salud pública. Iatreia. 2013;27(1):42-52.

4. Novoa JRU, Díaz GJ. Aflatoxinas: mecanismos de toxicidad. Rev Fac Med Univ Nac Colomb. 2006:54(2):108-116.

5. Kew MC. Aflatoxins as a cause of hepatocellular carcinoma. J Gastrointest Liver Dis JGLD. 2013;22(3):305-10.

6. Soussi T, Wiman KG. TP53: an oncogene in disguise. Cell Death Differ. 2015;22(8):1239-1249.

7. Aaltonen LA, Hamilton SR, World Health Organization, International Agency for Research on Cancer, editores. Pathology and genetics of tumours of the digestive 
system. Lyon: Oxford: IARC Press; Oxford University Press (distributor,); 2000. 314 p. (World Health Organization classification of tumours).

8. Villar S, Le Roux-Goglin E, Gouas DA, Plymoth A, Ferro G, Boniol M, et al. Seasonal Variation in TP53 R249S-Mutated Serum DNA with Aflatoxin Exposure and Hepatitis B Virus Infection. Environ Health Perspect. 2011;119(11):1635-40.

9. Hussain SP, Schwank J, Staib F, Wang XW, Harris CC. TP53 mutations and hepatocellular carcinoma: insights into the etiology and pathogenesis of liver cancer. Oncogene. 2007;26(15):2166-76.

10. Kew MC. Synergistic interaction between aflatoxin B1 and hepatitis B virus in hepatocarcinogenesis. Liver Int. 2003;23(6):405-409.

11. Yeh FS, Mo CC, Yen RC. Risk factors for hepatocellular carcinoma in Guangxi, People's Republic of China. Natl Cancer Inst Monogr. 1985;69:47-8.

12. Kirk GD, Lesi OA, Mendy M, Szymañska K, Whittle H, Goedert JJ, et al. 249(ser) TP53 mutation in plasma DNA, hepatitis B viral infection, and risk of hepatocellular carcinoma. Oncogene. 2005;24(38):5858-67.

13. Sell S, Hunt JM, Dunsford HA, Chisari FV. Synergy between hepatitis B virus expression and chemical hepatocarcinogens in transgenic mice. Cancer Res. 1991;51(4):1278-1285.

14. Bannasch P, Khoshkhou NI, Hacker HJ, Radaeva S, Mrozek M, Zillmann U, et al. Synergistic hepatocarcinogenic effect of hepadnaviral infection and dietary aflatoxin B1 in woodchucks. Cancer Res. 1995;55(15):3318-3330.

15. Pitzurra L, Fringuelli R, Perito S, Schiaffella F, Barluzzi R, Bistoni F, et al. A new azole derivative of 1,4-benzothiazine increases the antifungal mechanisms of natural effector cells. Antimicrob Agents Chemother. 1999;43(9):2170-5.

16. Aguilar F, Hussain SP, Cerutti P. Aflatoxin B1 induces the transversion of G $\rightarrow \mathrm{T}$ in codon 249 of the p53 tumor suppressor gene in human hepatocytes. Proc Natl Acad Sci. 1993;90(18):8586-8590.

17. Zhang W, He H, Zang M, Wu Q, Zhao H, Lu L, et al. Genetic Features of Aflatoxin-Associated Hepatocellular Carcinoma. Gastroenterology. 2017;153(1):249-262.e2.

18. Stern MC, Umbach DM, Mimi CY, London SJ, Zhang Z-Q, Taylor JA. Hepatitis B, aflatoxin B1, and p53 codon 249 mutation in hepatocellular carcinomas from Guangxi, People's Republic of China, and a meta-analysis of existing studies. Cancer Epidemiol Biomarkers Prev. 2001;10(6):617-625.

19. Galy O, Chemin I, Le Roux E, Villar S, Le Calvez-Kelm F, Lereau M, et al. Mutations in TP53 and CTNNB1 in Relation to Hepatitis B and C Infections in Hepatocellular Carcinomas from Thailand. Hepat Res Treat. 2011;2011:697162.

20. Lipigorngoson S, Limtrakul P, Suttajit M, Yoshizawa T. In-house direct cELISA for determining aflatoxin B 1 in Thai corn and peanuts. Food Addit Contam. 2003;20(9):838-45. 
21. Wigginton JE, Cutler DJ, Abecasis GR. A Note on Exact Tests of Hardy-Weinberg Equilibrium. Am J Hum Genet. 2005;76(5):887-93.

22. Nogueira JA, Ono-Nita SK, Nita ME, de Souza MM, do Carmo EP, Mello ES, et al. 249 TP53 mutation has high prevalence and is correlated with larger and poorly differentiated HCC in Brazilian patients. BMC Cancer [Internet]. 2009 [citado 11 de abril de 2017];9(1). Disponible en: http://bmccancer.biomedcentral.com/articles/10.1186/1471-2407-9-204

23. Lereau M, Gouas D, Villar S, Besaratinia A, Hautefeuille A, Berthillon P, et al. Interactions between hepatitis $B$ virus and aflatoxin B1: effects on p53 induction in HepaRG cells. J Gen Virol. 2012;93(3):640-50.

24. Liu Y, Wu F. Global Burden of Aflatoxin-Induced Hepatocellular Carcinoma: A Risk Assessment. Environ Health Perspect. 2010;118(6):818-24.

25. Navas M-C, Suarez I, Carreño A, Uribe D, Rios WA, Cortes-Mancera F, et al. Hepatitis B and Hepatitis C Infection Biomarkers and TP53 Mutations in Hepatocellular Carcinomas from Colombia. Hepat Res Treat. 2011;2011:1-10.

26. Soini Y, Chia SC, Bennett WP, Groopman JD, Wang JS, DeBenedetti VM, et al. An aflatoxin-associated mutational hotspot at codon 249 in the p53 tumor suppressor gene occurs in hepatocellular carcinomas from Mexico. Carcinogenesis. 1996;17(5):1007-12.

27. de Carvalho FM, de Almeida Pereira T, Gonçalves PL, Jarske RD, Pereira FEL, Louro ID. Hepatocellular carcinoma and liver cirrhosis TP53 mutation analysis reflects a moderate dietary exposure to aflatoxins in Espírito Santo State, Brazil. Mol Biol Rep. 2013;40(8):4883-7.

28. Contreras OLR, Flórez AMW. Determinación de aflatoxinas en alimentos de mayor consumo infantil comercializados en la ciudad de Pamplona, Norte de Santander. Bistua Rev Fac Cienc Básicas [Internet]. 2009 [citado 24 de febrero de 2016];7(1). Disponible en: http://www.redalyc.org/articulo.oa?id=90312171015

29. Morris Navarro LF, others. Determinación de aflatoxinas en muestras de maíz (Zea mays) y arroz (Oryza sativa) para consumo humano en cinco departamentos de la Costa Caribe Colombiana mediante cromatografía de alta eficiencia durante seis meses en 2011 [Internet]. Universidad Nacional de Colombia; 2011 [citado 24 de febrero de 2016]. Disponible en: http://www.bdigital.unal.edu.co/4908/

30. Diaz GJ, Krska R, Sulyok M. Mycotoxins and cyanogenic glycosides in staple foods of three indigenous people of the Colombian Amazon. Food Addit Contam Part B. 2015;150922031753004.

31. Grupo del Sistema de Análisis de Riesgos Químicos en Alimentos y Bebidas, Instituto Nacional de Vigilancia de Medicamentos y Alimentos INVIMA. Plan nacional subsectorial de vigilancia y control de aflatoxinas y conservantes en arepas para el año 2015 [Internet]. 2015 [citado 10 de marzo de 2016]. Disponible en: https:// www.invima.gov.co/images/pdf/inspecion y vigilancia/direccion-alimentos/ Documentotecnicoaflatoxinasyconservantesarepapublicar.pdf 
32. Resolución 02671 de 2014. Ministerio de Salud y Protección Social, 26712014.

33. Wu F, Khlangwiset P. Health economic impacts and cost-effectiveness of aflatoxin-reduction strategies in Africa: case studies in biocontrol and post-harvest interventions. Food Addit Contam Part A. 2010;27(4):496-509.

34. Yao J-G, Huang X-Y, Long X-D. Interaction of DNA repair gene polymorphisms and aflatoxin B1 in the risk of hepatocellular carcinoma. Int J Clin Exp Pathol. 2014;7(9):6231-44. 